\title{
Solid Solutions of Oxide Crystals as Substrates for Epitaxial Layer Depositions
}

\author{
M. Berkowski ${ }^{a, *}$, J. Fink-Finowicki ${ }^{a}$, R. AleksiJko ${ }^{a}$, \\ R. DiduszKo ${ }^{a, b}, \mathrm{P}$. BYsZewsKi ${ }^{a}$ \\ AND R. KiKALEJShViLi-DomukhovskA ${ }^{a}$ \\ ${ }^{a}$ Institute of Physics, Polish Academy of Sciences \\ al. Lotników 32/46, 02-668 Warsaw, Poland \\ ${ }^{b}$ Institute of Electronic Materials Technology \\ Wólczyńska 133, 01-919 Warsaw, Poland
}

\begin{abstract}
We present results of investigation on growth of solid solution crystals with perovskites and $\mathrm{K}_{2} \mathrm{NiF}_{4}$ structures used as substrates for epitaxy. Perovskite single crystals with no twins and crystals with $\mathrm{K}_{2} \mathrm{NiF}_{4}$ structure with the lattice parameter in the range $3.876-3.819 \AA$ and 3.754 to $3.688 \AA$, respectively, can be grown. Here preliminary results on investigation on growth of other solid solution crystals with the lattice constant from 3.946 to $3.688 \AA$ are also presented thus covering the whole interesting range for depositing oxide materials. These crystals can be grown by the Czochralski method that secures their high structural quality. Discussed crystals are resistant to reaction with the deposited oxide layers.
\end{abstract}

PACS numbers: 81.10.-h, 81.10.Fq, 61.10.Nz, 74.78.Bz

\section{Introduction}

Various oxide crystals are commonly used as substrates for thin layers epitaxial deposition of high temperature superconductors (HTSc) and manganese or cobalt-based perovskite exhibiting colossal magnetoresistance (CMR). Perovskites and pseudoperovskites with $\mathrm{K}_{2} \mathrm{NiF}_{4}$ structure belong to the most popular substrate materials.

*corresponding author; e-mail: berko@ifpan.edu.pl 


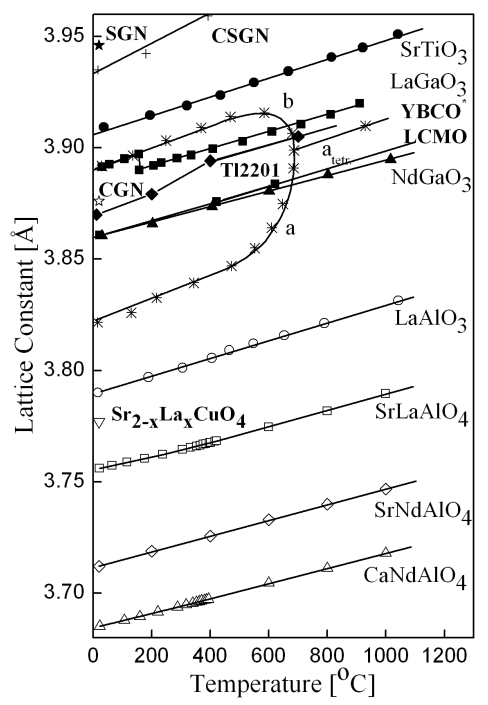

Fig. 1. Temperature dependence of the lattice constant of the most common high temperature superconductors, manganites, and typical substrates used for the epitaxy.

The substrate should have similar atoms distribution in the unit cell and comparable lattice constant with the mismatch not exceeding $0.2 \%$ in the temperature range from the epitaxy down to the application temperature. The appropriate lattice constant for deposition of various layers may be found among solid solution crystals. The comparison of the lattice constants of several HTSc and CMR perovskite with popular substrate materials within a wide temperature range is presented in Fig. 1.

Most of the materials with the perovskite structure undergoes temperature induced phase transitions between melting and room temperature. The phase transitions often causes defects such as twins in single crystals. Thus changing temperature during epitaxy process may lead to roughness of the substrates that leads to bending of the deposited layer. The perovskites with a cubic structure are especially suitable for substrates because they do not have any phase transition. There are also no phase transitions in materials with tetragonal $\mathrm{K}_{2} \mathrm{NiF}_{4}$ structure.

The substrate materials should have relatively high melting temperature. It is generally accepted that materials with the melting temperature above $1700^{\circ} \mathrm{C}$ are sufficiently stable towards reaction with most of the deposited layers. The melting temperature of the materials should not exceed $2100^{\circ} \mathrm{C}$ because of temperature limit of the crucibles used during crystals growth by the Czochralski method. This method allows to grow good quality crystals if they melt congruently.

\section{Perovskites}

The unit cell of the ideal not deformed $\mathrm{ABO}_{3}$ perovskite may be presented as a cube with larger $\mathrm{A}$ ions at the corners, smaller $\mathrm{B}$ ions in the center, and 
oxygen ions occupy centers of the cube's faces. At such an arrangement of the atoms in the unit cell oxygen ions form perfect octahedral lattice oriented along the crystallographic axes. It requires that radii of all ions fulfill the relation

$$
t=\frac{R_{\mathrm{A}}+R_{\mathrm{O}}}{\sqrt{2}\left(R_{\mathrm{B}}+R_{\mathrm{O}}\right)}=1,
$$

where $R_{\mathrm{A}}, R_{\mathrm{B}}$, and $R_{\mathrm{O}}$ are radii of $\mathrm{A}, \mathrm{B}$, and $\mathrm{O}$ ions, respectively.

In many perovskites ionic radii $R_{\mathrm{A}}$ and/or $R_{\mathrm{B}}$ do not fit to the ideal unit cell and the lattice undergoes deformation. Deviation from the ideal ionic radii is measured by parameter $t$ called a tolerance factor. With a decrease in the $R_{\mathrm{A}}$ radius volume of the perovskite unit lattice decreases and the oxygen octahedra become rotated and deformed losing their symmetry, then the space symmetry changes from the cubic $P m-3 m$ through tetragonal $P 4 / m b m$ or rhombohedral $R-3 c$ to the orthorhombic Pbnm one. Along with the deformation of the octahedra the cations A and B are shifted from the positions that they occupied in the ideal perovskite lattice. In strongly deformed lattices, distances between ion $\mathrm{A}$ and surrounding oxygen ions differ even by $0.7 \AA$, similarly distances B-O differ, thus coordination numbers and tolerance factor $t$ are not well defined.

Simple galate and aluminate rare earth perovskites form solid solution in a wide concentration range of components [1-6]. It was found that the concentration range decreases with increasing a difference in size of substitutes for A or B ions. The temperatures of the first and second order phase transition increase linearly with the concentration of the second component with decreasing the radius of ions substituted for La and temperatures decrease when the substituting ion is larger than La.

Interesting alternative in a search for new perovskite materials were experiments on growth of solid solutions of simple perovskites like $\mathrm{LaAlO}_{3}(\mathrm{LA})$ and $\mathrm{NdGaO}_{3}$ with perovskites with $\mathrm{Sr}$ at $\mathrm{A}$ sites and $\mathrm{Al}$ or Ta at $\mathrm{B}$ sites with stoichiometric formula $\mathrm{SrAl}_{0.5} \mathrm{Ta}_{0.5} \mathrm{O}_{3}$ (SAT) or $\mathrm{Al}$ and $\mathrm{Nb}$ that is $\mathrm{SrAl}_{0.5} \mathrm{Nb}_{0.5} \mathrm{O}_{3}$ (SAN) as the second component [7-13]. The first component has a deformed lattice while the second - a cubic one. It turned out that the solid solutions with up to $30 \%$ of the first component melt congruently and maintained the cubic lattice. The solid solutions based on SAT and LA [9-13] proved the most stable, therefore most intensively investigated.

As a further development of investigations of perovskite solid solutions may be an exploration of the ternary system where as the third component is perovskite $\mathrm{CaAl}_{0.5} \mathrm{Ta}_{0.5} \mathrm{O}_{3}$ (CAT) with the orthorhombic Pbnm structure [14]. Composition of the melt used for crystal growth are shown in the composition triangle in Fig. 2.

The SAT:LA solid solutions (on the SAT-LA line in Fig. 2) were investigated in the whole composition range. It was found that they exist and adopt cubic structure within $0 \%$ to $50 \%$ LA range, however the crystals may be grown by the Czochralski method only in the range approximately $23-43 \mathrm{~mol} \%$ of LA. The single crystals adopt the cubic structure with a mixture of two cubic phases: of the 


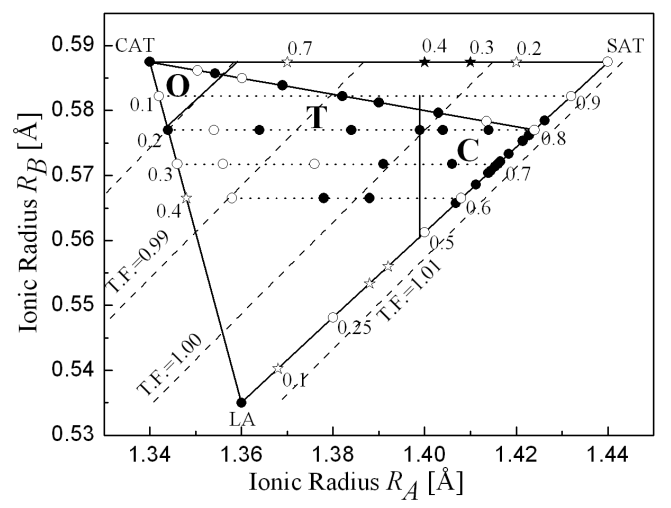

Fig. 2. Compositions of the crystals shown as a function of the $R_{\mathrm{A}}$ and $R_{\mathrm{B}}$ ionic radii with the calculated tolerance factor (T.F.) - broken lines and boundaries between (O) orthorhombic, (T) tetragonal, and (C) cubic structures — solid lines; - — crystals grown by the Czochralski method; o — crystals grown by the floating zone method; full stars - the melt compositions from which the multiphase boules were grown by the Czochralski method; open stars - the rod compositions from which the multiphase boules were grown by the floating zone method.

$P m-3 m$ symmetry with simple perovskite unit cell and of $F m-3 m$ symmetry with the unit cell built of 8 simple perovskite cells. The two phases have the same average composition but in the $F m-3 m$ phase the $\mathrm{Al}$ and $\mathrm{Ta}_{1-x} \mathrm{Al}_{x}$ cations are ordered within the unit cell. In SAT:LA solid solutions the amount of the ordered structure of $\mathrm{Al}$ and $\mathrm{Ta}_{1-x} \mathrm{Al}_{x}$ ions decreases with adding $\mathrm{LA}$ and totally disappears at $40 \%$ of LA in the crystals. The dimensions of the ordered regions are of the order of $200 \AA$.

With decreasing ionic radii $R_{\mathrm{A}}$ and $R_{\mathrm{B}}$ in the ternary systems the crystallographic structure changes from the cubic $P m-3 m$ and $F m-3 m$ through tetragonal $P 4 / m b m$ to the orthorhombic Pbnm symmetry. The ternary system allows to extend the range of the cubic lattice constant from 3.876-3.850 $\AA$ in $\mathrm{SAT}_{1-x} \mathrm{LA}_{x}$ and to $3.843 \AA$ if $\mathrm{NdAlO}_{3}$ (NA) is substituted for LA [8]. Because there were not discovered any low angle twins in crystals with the tetragonal structure therefore the useful range of the lattice parameters might be further extended down to $3.819 \AA$.

The lowest values of the lattice constants in the ternary system were found in the CAT:LA crystals at the CAT-LA line in Fig. 2. Substitution of NA for LA further decreases the lattice constant. The $\mathrm{CAT}_{1-x} \mathrm{NA}_{x}$ crystals with $x$ from 0.5 to 0.8 have the rhombohedral $R-3 c$ structure and the lattice constant falling to $3.77 \AA$. The materials exhibit the second order phase transitions thus it seems improbable to obtain crystals without any twins.

Other interesting substrate materials with the lattice constant covering the range 3.79-3.75 $\AA$ are LA solid solutions with $\mathrm{NA}$ or $\mathrm{PrAlO}_{3}$. They form the solid 


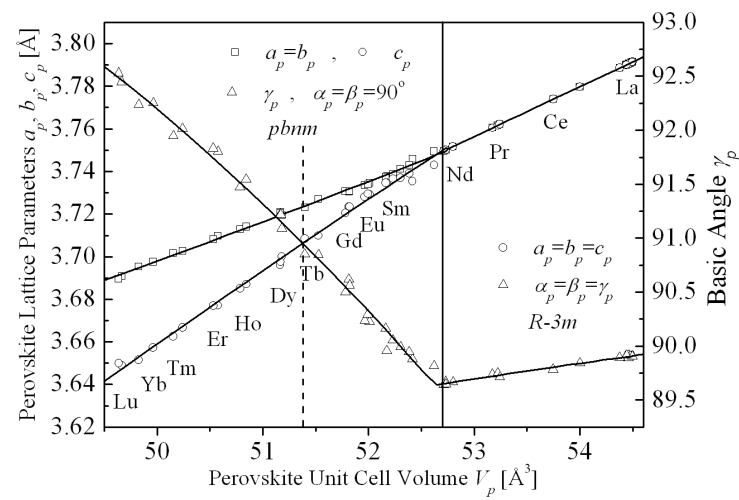

Fig. 3. Rare earth aluminum perovskites lattice parameters vs. perovskite unit cell volume.

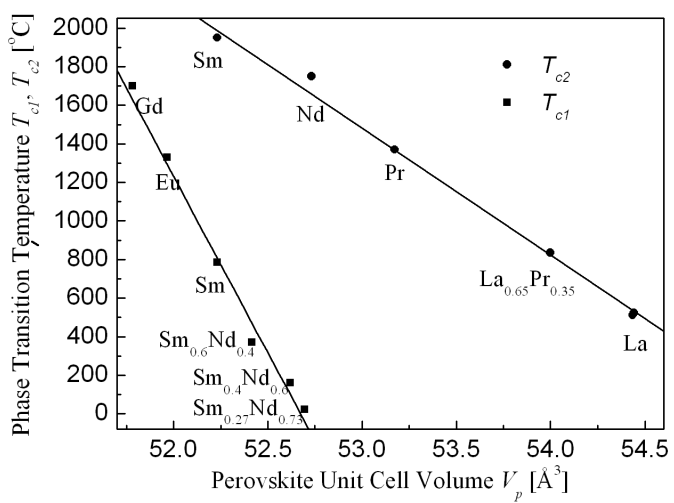

Fig. 4. First and second order phase transition temperatures $\left(T_{\mathrm{c} 1}, T_{\mathrm{c} 2}\right)$ in rare earth aluminum perovskites vs. perovskite unit cell volume.

solutions in whole concentration range, melt congruently and may be grown by the Czochralski method; the crystals adopt rhombohedral $R-3 c$ structure. The published data allow one to find the dependence of the perovskite lattice parameters as a function of the perovskite unit cell volume which is shown in Fig. 3. It results from an interpolation that $\mathrm{LA}_{1-x} \mathrm{NA}_{x}$ at $x \approx 0.3$ should have zero mismatch toward $\mathrm{Sr}_{2-x} \mathrm{La}_{x} \mathrm{CuO}_{4}$ superconductor. The phase transition temperature dependence on the perovskite unit cell volume shown in Fig. 4 proves that the phase transition in this substrate material occurs at $\approx 900^{\circ} \mathrm{C}$ that is above the epitaxy process temperature. The only disadvantage of the materials is high melting point $2080-2100^{\circ} \mathrm{C}$ of the components.

The lattice constant range from 3.88 to almost $3.95 \AA$ is covered by perovskites where a mixture of $\mathrm{Sr}$ and $\mathrm{Ca}$ occupies $\mathrm{A}$ sites and $\mathrm{Ga}$ and $\mathrm{Nb}$ - the $\mathrm{B}$ sites [15]. The calcium perovskite $\mathrm{CaGa}_{0.5} \mathrm{Nb}_{0.5} \mathrm{O}_{3}$ (CGN) has the orthorhombic Pbnm structure and the strontium $\mathrm{SrGa}_{0.5} \mathrm{Nb}_{0.5} \mathrm{O}_{3}$ (SGN) the cubic with the 
mixture of Pm-3m and Fm-3m phases. They form solid solutions at some composition with the cubic structure and the lattice constant $\approx 3.94 \AA[15]$. Both materials are strongly reduced during melting in the oxygen-free atmosphere and the crystals are not transparent; annealing them for a few hours in oxidizing atmosphere at a temperature about $1000^{\circ} \mathrm{C}$ turns them to light-yellow and transparent. The growth processes proved existence of the solid solutions at any composition. The effective segregation coefficient $\mathrm{Ca} / \mathrm{Sr}$ is close to 0.43 and $\mathrm{Ga} / \mathrm{Nb}$ is negligible $\approx 0.94$. The $\mathrm{SGN}_{1-x} \mathrm{CGN}_{x}$ solid solutions adopt the cubic structure at $x>0.6$ with the lattice constant range from 3.924 to $3.495 \AA$. At lowering Sr content the crystals have at first the tetragonal $\mathrm{P} 4 / \mathrm{mbm}$ and later orthorhombic $\mathrm{Pbnm}$ structure.

\section{Materials with tetragonal $\mathrm{K}_{2} \mathrm{NiF}_{4}$ structure}

Single crystals with the tetragonal $\mathrm{K}_{2} \mathrm{NiF}_{4}$ structure do not exhibit any phase transitions, they are attractive because there are not any perovskites without phase transitions with the lattice constant from $3.843 \AA$ in $\mathrm{SrLaGaO}_{4}$ (SLG) to $3.688 \AA$ in $\mathrm{CaNdAlO}_{4}$. The aluminates melt congruently and the galates incongruently with a peritectic transition, however both of them can be grown by the Czochralski method. The most interesting would be the material with the lattice constant $3.777 \AA$ suitable for $\mathrm{Sr}_{2-x} \mathrm{La}_{x} \mathrm{CuO}_{4}$ superconductor. The lattice constant $3.754 \AA$ of $\mathrm{SrLaAlO}_{4}$ (SLA) is the closest to this value. The crystallization of $\mathrm{SLA}_{1-x} \mathrm{SLG}_{x}$ solid solutions were investigated $[16,17]$ and it was shown that gallium may be substituted for aluminum up to $x=0.27$. The quality of the crystals drastically lowers with increasing $x$ because one of the components of the solid solution melts incongruently. Similar problems were encountered during crystallization of $\mathrm{SLA}_{1-x}\left(\mathrm{Sr}_{2} \mathrm{Al}_{0.5} \mathrm{Ta}_{0.5} \mathrm{O}_{4}\right)_{x}$ solid solutions.

It is considerably simpler to obtain the solid solutions with the $\mathrm{K}_{2} \mathrm{NiF}_{4}$ structure and with the lattice constants in the range 3.754-3.688 $\AA$. The first part of the range covers the $\mathrm{SLA}_{1-x}\left(\mathrm{SrNdAlO}_{4}\right)_{x}$ solid solutions and single crystals were grown by the Czochralski method. These materials are similar to $\left(\mathrm{SrNdAlO}_{4}\right)_{1-x}\left(\mathrm{CaNdAlO}_{4}\right)_{x}$ that were investigated by Novoselov et al. [18]. Thus growth of these materials depends only on the need of substrates with the specified lattice parameters.

\section{Conclusions}

Search for new crystals leads us to investigate various materials with perovskites and $\mathrm{K}_{2} \mathrm{NiF}_{4}$ structure that are interesting substrate materials. Because these materials are the solid solutions the lattice constant can be varied in wide range which allows to match substrate to specific epitaxial layer. Good quality single crystals without twins can be grown with the lattice constant from 3.876 to $3.819 \AA$ with perovskite structure and in the range from 3.754 to $3.688 \AA$ with the $\mathrm{K}_{2} \mathrm{NiF}_{4}$ structure. The crystals are chemically stable at the epitaxy temperature, they have low dielectric constant $\varepsilon$ and low loss coefficient $\tan \delta$. 


\section{Acknowledgments}

This work was partially supported by the State Committee for Scientific Research under grant No. 2 P03B 04423.

\section{References}

[1] M. Berkowski, J. Fink-Finowicki, W. Piekarczyk, L. Perchuć, P. Byszewski, L.O. Vasylechko, D.I. Savytskij, K. Mazur, J. Sass, E. Kowalska, J. Kapuśniak, J. Cryst. Growth 209, 75 (2000).

[2] M. Berkowski, J. Fink-Finowicki, P. Byszewski, R. Diduszko, E. Kowalska, R. Aleksiyko, W. Piekarczyk, L.O. Vasyleczko, D.I. Savytskij, L. Perchuć, J. Kapuśniak, J. Cryst. Growth 222, 194 (2001).

[3] R. Aleksiyko, M. Berkowski, P. Byszewski, B. Dạbrowski, R. Diduszko, J. Fink-Finowicki, L.O. Vasylechko, Cryst. Res. Technol. 36, 789 (2001).

[4] A. Yoshikawa, A. Saitow, H. Horiuchi, T. Shishido, T. Fukuda, J. Alloys Comp. 266, 104 (1998).

[5] P.D. Dernier, R.G. Maines, Mater. Res. Bull. 6, 433 (1971).

[6] S.J. Schneider, R.S. Roth, J.L. Waring, J. Res. Natl. Bur. Standards 65A, 345 (1961).

[7] C.D. Brandle, V.J. Fratello, J. Mater. Res. 5, 2160 (1990).

[8] D. Mateika, H. Köhler, H. Laudan, E. Völkel, J. Cryst. Growth 109, 447 (1991).

[9] K. Shimamura, H. Tabata, H. Takeda, V.V. Kochurikhin, T. Fukuda, J. Cryst. Growth 194, 209 (1998).

[10] M. Ito, K. Shimamura, D.A. Pawlak, T. Fukuda, J. Cryst. Growth 235, 277 (2002)

[11] M. Steins, J. Doerschel, P. Reiche, Z. Kristallogr. 212, 77 (1997).

[12] J. Xiao, M. Shao, Y. Tian, W. Huang, A. Wang, S. Yin, J. Cryst. Growth 326, 671 (2002).

[13] M. Berkowski, J. Fink-Finowicki, R. Diduszko, P. Byszewski, R. Aleksiyko, R. Kikalejshvili-Domukhovska, J. Cryst. Growth 257, 146 (2003).

[14] M. Berkowski, R. Aleksiyko, J. Fink-Finowicki, R. Diduszko, P. Byszewski, R. Kikalejshvili-Domukhovska, J. Cryst. Growth 269, 512 (2004).

[15] S. Erdei, L.E. Cross, F.W. Ainger, A. Bhalla, J. Cryst. Growth 139, 54 (1994).

[16] M. Berkowski, J. Alloys Comp. 251, 1 (1997).

[17] M. Berkowski, J. Fink-Finowicki, E. Mojaiskaia, Acta Phys. Pol. A 92, 182 (1997).

[18] A. Novoselov, M. Ryumin, G. Pushkina, F. Spirdonov, G. Zimina, L. Komissarova, A. Pajączkowska, Cryst. Res. Technol. 40, 405 (2005). 